University of Windsor

Scholarship at UWindsor

1991

\title{
A unified treatment of the non-relativistic and relativistic hydrogen atom I: The wavefunctions
}

\author{
R. A. Swainson \\ Gordon W. F. Drake \\ University of Windsor
}

Follow this and additional works at: https://scholar.uwindsor.ca/physicspub

Part of the Physics Commons

\section{Recommended Citation}

Swainson, R. A. and Drake, Gordon W. F.. (1991). A unified treatment of the non-relativistic and relativistic hydrogen atom I: The wavefunctions. Journal of Physics A: Mathematical and General, 24 (1), 79-94.

https://scholar.uwindsor.ca/physicspub/34 
A unified treatment of the non-relativistic and relativistic hydrogen atom I: The wavefunctions

This article has been downloaded from IOPscience. Please scroll down to see the full text article.

1991 J. Phys. A: Math. Gen. 2479

(http://iopscience.iop.org/0305-4470/24/1/019)

View the table of contents for this issue, or go to the journal homepage for more

Download details:

IP Address: 137.207.184.30

The article was downloaded on 26/04/2013 at 02:41

Please note that terms and conditions apply. 


\title{
A unified treatment of the non-relativistic and relativistic hydrogen atom I: the wavefunctions
}

\author{
Robin A Swainson and G W F Drake \\ Department of Physics, University of Windsor, Windsor, Ontario, Canada N9B 3P4
}

Received 26 June 1990

\begin{abstract}
In this and the following two papers in this series it is shown how the radial part of non-relativistic and relativistic hydrogenic bound-state calculations involving the Green functions can be presented in a unified manner. The angular part of such calculations, being well understood, is performed in the standard way. In this, the first paper, it is shown how a suitable linear transformation of the two relativistic radial wavefunctions allows the pair of relativistic coupled differential equations to be written as two uncoupled second. order equations which are simple generalizations of the corresponding non-relativistic equation. This transformation is presented in a manner which allows for a simple extension to the Green function problem. The transformed relativistic wavefunctions are explicitly derived and the normalization is presented in a novel and simple way. A new derivation is given for the recursion relations for both non-relativistic and relativistic radial wavefunctions, some of which are new. These relations are required in the subsequent papers.
\end{abstract}

\section{Introduction}

The importance of the part played by the Green functions in the analysis of spectroscopic properties of hydrogenic ions hardly needs emphasizing [1]. Indeed the analysis of two-photon processes in such systems inevitably requires the calculation of matrix elements of the appropriate hydrogenic Green function. Although several non-relativistic calculations employing the Schrödinger-Coulomb Green function (SCGF) have been performed over the years, the corresponding relativistic calculations have received considerably less attention. This is probably because in the standard representations both the Dirac-Coulomb wavefunction (DCWF) and the Dirac-Coulomb Green function (DCGF) are rather hard to deal with: the angular dependence is given by four-component Dirac spinors, and the general radial dependence consists of terms involving several separate functions.

Since relativistic calculations are of value, however, there has been a continuing interest in the development of approximate methods designed to circumvent the difficulties encountered in an exact treatment. Of the several approximate methods which have been used, probably the best known is the representation of the Green function by a variationally determined finite basis set [2]. Describing as it does the simplest relativistic atomic system, the Dirac equation for the hydrogen atom is of course completely soluble. We thus find ourselves in the curious situation wherein the methods being applied to problems one would expect to admit of exact analytical solutions are almost exclusively approximate. Thus relativistic hydrogenic two-photon processes such as two-photon bound-bound transitions, two-photon ionization, Raman scattering, and even the Lamb shift have been treated by various approximate techniques 
even though there surely exist exact solutions. Similarly, exact calculations of relativistic second-order perturbation effects, requiring the use of the reduced Dirac-Coulomb Green function (RDCGF), are possible in many cases and yet have not been extensively analysed.

In this series of papers $[3,4]$ we attempt to remedy this situation by presenting a framework for the simplified treatment of calculations involving the relativistic DCGF and RDCGF. Our simplification consists in fact of a unification of the non-relativistic and relativistic calculations into one general form. Once the non-relativistic calculations have been performed, the way will be clear for a fully relativistic calculation.

Powerful methods are currently available for the treatment of the angular part of specific calculations of matrix elements of the Green functions [5] and are, in our view, worth retaining. Our work then focuses almost exclusively on the radial properties of the Green functions, and indeed the unification of non-relativistic and relativistic theory is achieved for the radial functions.

Our treatment of both the DCWF and the DCGF is entirely conventional at the outset, with the standard separation into an angular part (represented by four-component Dirac spinors) and a radial part [6]. The radial part of the DCWF consists of two radial functions satisfying two coupled first-order differential equations, while that of the DCGF consists of four functions satisfying four coupled first-order differential equations. We have found simple linear transformations of the respective radial functions such that the transformed functions satisfy uncoupled second-order differential equations which are obvious generalizations of those satisfied by the radial part of the corresponding non-relativistic functions. The transformation also allows us to derive various properties of the radial DCWF and DCGF which are hidden in the standard representation. Our analysis will be seen to be applicable to the reduced DCGF also.

The transformation we rediscovered and which we present here was actually given for the wavefunctions many years ago by Infeld [7], though in a slightly different form. Infeld's discovery, presented in the context of a study of the factorization method, seems to have been largely overlooked in the light of a rather different approach to the unification of relativistic and non-relativistic theories of hydrogenic ions. This approach, used by Martin and Glauber [8] to derive both the DCWF and DCGF, is based on the second-order Dirac equation, which, in contrast to Infeld's second-order radial equations, is three dimensional. The solutions of the actual (first-order) equation are obtained from the solutions of the second-order equation by the application of a three-dimensional projection operator. Biedenharn [9] discovered a transformation of the second-order equation which decouples it into two non-relativistic-like equations, which are nonetheless still three dimensional. (Biedenharn's work was concerned with the continuum wavefunctions; the application of his transformation to the bound states was given by Wong and Yeh [10].) It was noticed only recently by Su [11] that Biedenharn's transformation could be applied directly to the first-order threedimensional Dirac equation with similar consequences. In this respect $\mathrm{Su}$ comes closest of all to reproducing Infeld's method.

Our approach differs then in several respects from other attempts at a unified treatment of the non-relativistic and relativistic hydrogen atom. We retain the standard angular analysis, concentrating on unifying the radial part of the theory. Thus it is the calculation of radial matrix elements of the Green functions which is considerably simplified by our method. In contrast, the approach based on the second-order Dirac equation requires a non-standard approach to the full three-dimensional integrals, and thus obscures the relatively simple angular analysis. 
We present our unified treatment in three papers $[3,4]$. This, the first paper, contains a detailed analysis of the wavefunctions and their recursion properties. Though much of the work presented here can be deduced from Infeld's paper we feel it important to reiterate the method for several reasons. First, Infeld's paper, not being directly concerned with the DCWFs is not specific enough for our purposes, which are directed towards applications of the theory. Our transformation of the DCWFs is slightly different from Infeld's, and since we generalize it in the subsequent papers on the Green functions, we feel it important to demonstrate the rationale behind its introduction in section 2 . In appendix 1 we present a novel method of solving the second-order differential equation using Laplace transforms, which leads to a remarkably simple and largely algebraic treatment of the recursion relations satisfied by the radial wavefunctions, given in section 3 , and also admits a simple generalization to the solution of the defining equation of the radial Green functions. The recursion relations will be found to be crucial to the ensuing work, particularly for our treatment of the reduced Coulomb Green functions. Finally, some aspects of the work such as the normalization integrals (appendix 2) differ markedly from Infeld's. (The normalization of Infeld's solution was given by Lin [12].)

In the second paper of this series [3] we give a detailed analysis of the SCGF and DCGF, the latter treated using a generalization of the transformation of the radial wavefunctions given in the first paper. We include a comprehensive presentation of various representations of the two Green functions, and their recursion relations, matrix elements and recursion relations of matrix elements are also examined.

We turn our attention to the reduced Green functions (RSCGF and RDCGF), the Green functions evaluated at an energy eigenvalue, in the third and final paper [4]. Our transformation of the radial functions is equally useful for the analysis of these special Green functions, required in the theory of second-order perturbations. Again we also discuss the matrix elements, as well as recursion relations of the functions and their matrix elements.

In a further paper we will demonstrate the utility of our method by presenting a completely analytical and exact calculation of the relativistic polarizability of hydrogenic ions.

\section{The Schrödinger- and Dirac-Coulomb equations}

The non-relativistic hydrogenic eigenfunctions $\psi_{E}(r)$ and corresponding energy eigenvalues $E$ are found by solving the Schrödinger-Coulomb equation [6],

$$
\left[-\frac{\hbar^{2}}{2 m} \nabla^{2}-\frac{\hbar^{2}}{a m r}-E\right] \psi_{E}(\boldsymbol{r})=0
$$

subject to the boundary condition that $\psi_{E}$ be a square integrable function, $\psi_{E} \in L^{2}\left(\mathbb{R}^{3}\right)$. (The scaled Bohr radius $a$ is related to the Bohr radius $a_{0}$ by $a=a_{0} / Z$.) As is well known, this equation is most naturally solved by transforming from cartesian to spherical polar coordinates, in which system it is completely separable. In fact, introducing the dimensionless 'generalized principal quantum number' $\nu$, defined by $\nu^{2} a^{2}=$ $-\hbar^{2} / 2 m E$, solutions to (2.1) are found to be of the form

$$
\psi_{E}(r, \theta, \phi)=R_{v \prime}(r) Y_{l m}(\theta, \phi)
$$

where the $Y_{l m}$ 's are spherical harmonics (we adopt Condon and Shortley's definitions 
and phase conventions) and $R_{v l}$, the radial part of the wavefunction, satisfies

$$
\left[\frac{1}{r^{2}} \frac{\mathrm{d}}{\mathrm{d} r}\left(r^{2} \frac{\mathrm{d}}{\mathrm{d} r}\right)-\frac{l(l+1)}{r^{2}}+\frac{2}{a r}-\frac{1}{\nu^{2} a^{2}}\right] R_{\nu l}(r)=0 .
$$

subject to the conditions that

$$
r R_{\nu l} \in L^{2}(\mathbb{R}) \quad \text { and } \quad \lim _{r \rightarrow 0} r R_{\nu l}(r)=0 .
$$

On solving this equation it is found that $\nu=n=l+1, l+2, \ldots$, an integer, and thus

$$
R_{n l}(r)=N_{n r}(2 r / a n)^{l} \mathrm{e}^{-r / a n} L_{n-l-1}^{2 l+1}(2 r / a n) .
$$

We chose $N_{n l}$ so as to normalize $R$,

$$
\int\left|\psi_{E}(r)\right|^{2} \mathrm{~d} r=\int_{0}^{\infty} r^{2} R_{n l}(r)^{2} \mathrm{~d} r=1
$$

Thus, taking note of the integral derived in appendix 2 (A2.2), we find

$$
N_{n l}=\left(2 / n^{2} a^{3 / 2}\right) \sqrt{(n-l-1) ! /(n+l) !} .
$$

An arbitrary phase factor may be introduced into the normalization constants; the only effect will be different signs in the recursion relations. The Schrödinger-Coulomb wavefunctions are thus completely determined by equation (2.5).

Although several methods of solving (2.3) are known, in appendix 1 we briefly sketch a solution using the method of Laplace transforms which can be adapted usefully for the solution of the corresponding Green function problem [3]. This solution is noteworthy for two reasons. First, it is equally well applicable to equations with non-integral 'angular momentum' eigenvalues, such as will arise shortly in the context of the radial Dirac-Coulomb wavefunctions. Secondiy, in the Laplace transformed space the differential equation to be solved is of first order and is thus relatively easy to deal with, once a particularly useful change of variables has been made. This solution is then very easily generalized to treat the defining radial differential equation of the Green functions, where we encounter an additional complication arising from the presence of a delta function. (See the following papers in this series [3,4].)

The relativistic hydrogenic eigenfunctions $\psi_{E}(\vec{r})$ (which are four-component spinors) and corresponding energy eigenvalues $E$ are found by solving the DiracCoulomb equation [6],

$$
\left[-\mathrm{i} \hbar c \boldsymbol{\alpha} \cdot \boldsymbol{\nabla}+\beta m c^{2}-\alpha Z \hbar c / \boldsymbol{r}-E\right] \psi_{E}(\boldsymbol{r})=0
$$

subject to the boundary condition that $\psi_{E}^{*} \psi_{E} \in L\left(\mathbb{R}^{3}\right)$. (Notation and special functions used in this paper are defined in appendix 3.) As with the Schrödinger-Coulomb equation, the natural method of solution is in spherical polar coordinates, though some modifications are required to take into account the four-component spinors. In keeping with the approach we have outlined in the introduction the present method of solution will follow the conventional separation into an angular and a radial part.

Defining [9] $K=\beta(\Sigma \cdot L(\theta, \phi)+\hbar)(2.8)$ becomes

$$
\left\{(\boldsymbol{\alpha} \cdot \hat{\boldsymbol{r}})\left[-\frac{\mathrm{i} h c}{r} \frac{\bar{d}}{\partial r} r+\frac{\mathrm{i} c}{r} \beta K\right]+\beta m c^{2}-\frac{\alpha \boldsymbol{Z}}{r}-E\right\} \psi_{E}(r, \theta, \phi)=0 .
$$

The total angular momentum operator, $\boldsymbol{J}(\theta, \phi)=\boldsymbol{L}(\theta, \phi)+\hbar / 2 \Sigma$ and $K$ commute with the Dirac-Coulomb operator; there are therefore solutions of $(2.9)$ which are eigenfunctions of $J^{2}, J_{z}$ and $K$, with eigenvalues $\hbar^{2} j(j+1), \hbar M$ and $-\hbar \kappa$ respectively. The 
allowed values, given the boundary conditions, are: $j=\frac{1}{2}, \frac{3}{2}, \ldots ; M=-j,-j+1, \ldots,+j$; $\kappa= \pm\left(j+\frac{1}{2}\right)$.

In view of this, solutions of (2.9) can be chosen such that

$$
\psi_{E}(r, \theta, \phi)=\left(\begin{array}{c}
f_{1}(r) \chi_{\kappa}^{\mathrm{M}}(\theta, \phi) \\
\mathrm{i} f_{2}(r) \chi_{-\kappa}^{\mathrm{M}}(\theta, \phi)
\end{array}\right)
$$

where

$$
\chi_{\kappa}^{\mathrm{M}}(\theta, \phi)=\left[\begin{array}{c}
\frac{-\kappa}{|\kappa|}\left(\frac{\kappa+1 / 2-M}{2 \kappa+1}\right)^{1 / 2} Y_{\left|\kappa+\frac{1}{2}\right|-\frac{1}{2}, \mathrm{M}-\frac{1}{2}}(\theta, \phi) \\
\left(\frac{\kappa+1 / 2+M}{2 \kappa+1}\right)^{1 / 2} Y_{\left|\kappa+\frac{1}{2}\right|-\frac{1}{2}, \mathrm{M}+\frac{1}{2}}(\theta, \phi)
\end{array}\right]
$$

and the radial wavefunctions satisfy

$$
\begin{aligned}
& {\left[m c^{2}-\frac{\alpha Z \hbar c}{r}-E\right] f_{1}(r)+\left[\frac{\hbar c \kappa}{r}-\frac{\hbar c}{r} \frac{\mathrm{d}}{\mathrm{d} r} r\right] f_{2}(r)=0} \\
& {\left[\frac{\hbar c}{r} \frac{\mathrm{d}}{\mathrm{d} r} r+\frac{\hbar c \kappa}{r}\right] f_{1}(r)-\left[m c^{2}+\frac{\alpha Z \hbar c}{r}+E\right] f_{2}(r)=0 .}
\end{aligned}
$$

The integrability condition on $\psi_{E}$ now implies that $\left|f_{1}\right|$ and $\left|f_{2}\right|$ be square integrable, and this is the main boundary condition we employ in solving $(2.12 a, b)$. In fact,

$$
\int \psi_{E}(\boldsymbol{r})^{\dagger} \psi_{E}(\boldsymbol{r}) \mathrm{d} \boldsymbol{r}=\int\left(\left|f_{1}(r)\right|^{2}+\left|f_{2}(r)\right|^{2}\right) r^{2} \mathrm{~d} r
$$

So far our treatment of the Dirac-Coulomb problem has been entirely conventional. In fact, throughout this work we shall be treating the angular part of the analysis in the standard manner. However, in our treatment of the radial part of the analysis we depart from the traditional route [1]. Thus we will solve the two radial equations (2.12) in a manner which can be shown to be equivalent to that proposed by Infeld [7]. Let

$$
\left(\begin{array}{l}
g_{1}(r) \\
g_{2}(r)
\end{array}\right)=\left(\begin{array}{cc}
1 & X \\
X & 1
\end{array}\right)\left(\begin{array}{l}
f_{1}(r) \\
f_{2}(r)
\end{array}\right)
$$

with $X$ to be determined. Then

$$
\left[\left(\varepsilon_{0}-\varepsilon\right)-\frac{\alpha Z+X \kappa-X}{r}+X \frac{\mathrm{d}}{\mathrm{d} r}\right] g_{1}(r)+\left[-X\left(\varepsilon_{0}-\varepsilon\right)+\frac{X \alpha Z+\kappa-1}{r}-\frac{\mathrm{d}}{\mathrm{d} r}\right] g_{2}(r)=0
$$

and

$$
\left[X\left(\varepsilon_{0}+\varepsilon\right)+\frac{X \alpha Z+\kappa+1}{r}+\frac{\mathrm{d}}{\mathrm{d} r}\right] g_{1}(r)-\left[\left(\varepsilon_{0}+\varepsilon\right)+\frac{\alpha Z+X \kappa+X}{r}+X \frac{\mathrm{d}}{\mathrm{d} r}\right] g_{2}(r)=0
$$

and, on eliminating a derivative from each equation in turn,

$$
\begin{aligned}
{\left[\varepsilon_{0}\left(1-X^{2}\right)-\right.} & \left.\varepsilon\left(1+X^{2}\right)-\frac{\alpha Z\left(1+X^{2}\right)+2 X \kappa}{r}\right] g_{1}(r) \\
& +\left[2 X \varepsilon+\frac{2 X \alpha Z+\left(1+X^{2}\right) \kappa-\left(1-X^{2}\right)}{r}-\left(1-X^{2}\right) \frac{\mathrm{d}}{\mathrm{d} r}\right] g_{2}(r)=0
\end{aligned}
$$




$$
\begin{aligned}
& {\left[2 X \varepsilon+\frac{2 X \alpha Z+\left(1+X^{2}\right) \kappa+\left(1-X^{2}\right)}{r}+\left(1-X^{2}\right) \frac{\mathrm{d}}{\mathrm{d} r}\right] g_{1}(r)} \\
& -\left[\varepsilon_{0}\left(1-X^{2}\right)+\varepsilon\left(1+X^{2}\right)+\frac{\alpha Z\left(1+X^{2}\right)+2 X \kappa}{r}\right] g_{2}(r)=0 .
\end{aligned}
$$

Here, $\varepsilon=E / \hbar c$ and $\varepsilon_{0}=m c^{2} / \hbar c$. Quite remarkably, if we now choose

$$
X=(-\kappa+\gamma) / \alpha Z
$$

where $\gamma=\sqrt{\kappa^{2}-\alpha^{2} Z^{2}}$, one term in each of the two equations drops out, and we are left with

$$
\begin{aligned}
& {\left[\varepsilon_{0}-\frac{\varepsilon \kappa}{\gamma}\right] g_{1}(r)+\left[-\frac{\alpha Z \varepsilon}{\gamma}+\frac{\gamma-1}{r}-\frac{\mathrm{d}}{\mathrm{d} r}\right] g_{2}(r)=0} \\
& {\left[-\frac{\alpha Z \varepsilon}{\gamma}+\frac{\gamma+1}{r}+\frac{\mathrm{d}}{\mathrm{d} r}\right] g_{1}(r)-\left[\varepsilon_{0}+\frac{\varepsilon \kappa}{\gamma}\right] g_{2}(r)=0 .}
\end{aligned}
$$

Finally, we eliminate $g_{1}$ and $g_{2}$ in turn from the equations to arrive at the form we desire:

$$
\begin{aligned}
& {\left[\frac{\mathrm{d}^{2}}{\mathrm{~d} r^{2}}+\frac{2}{r} \frac{\mathrm{d}}{\mathrm{d} r}-\frac{\gamma(\gamma+1)}{r^{2}}+\frac{2 \alpha Z \varepsilon}{r}-\omega^{2}\right] g_{1}(r)=0} \\
& {\left[\frac{\mathrm{d}^{2}}{\mathrm{~d} r^{2}}+\frac{2}{r} \frac{\mathrm{d}}{\mathrm{d} r}-\frac{(\gamma-1) \gamma}{r^{2}}+\frac{2 \alpha Z \varepsilon}{r}-\omega^{2}\right] g_{2}(r)=0}
\end{aligned}
$$

where $\omega=\sqrt{\varepsilon_{0}^{2}-\varepsilon^{2}}$. Solutions of (2.19) do not necessarily satisfy (2.18); the relative values of the normalization factors have to be choser correctly. Thus equations (2.18) are, strictly speaking, equivalent to $(2.19 a)$ and $(2.18 b)$ or $(2.19 b)$ and $(2.18 a)$.

Since $L^{2}(\mathbb{R})$ is a linear space the boundary conditions on $g_{1}$ and $g_{2}$ are the same as those on $f_{1}$ and $f_{2}$. Thus:

$$
r g_{1}, r g_{2} \bullet L^{2}(\mathbb{R}) \quad \lim _{r \rightarrow 0} r g_{1}(r), r g_{2}(r)=0 .
$$

Equations (2.19a) and $(2.19 b)$ are simply generalizations of the defining equation for the radial Schrödinger-Coulomb wavefunction, (2.3). The principal difference, of course, is that $\gamma$, unlike $l$, is non-integral. However, as we show in appendix 1 , this fact is not significant as far as the form of the solutions is concerned. Indeed, invoking at this point the theorem presented in appendix 1 , we can immediately state that the solutions to $(2.19 a)$ and $(2.19 b)$ are

$$
\begin{aligned}
& g_{1}^{\mathrm{N} \kappa}(r)=N_{1}\left(2 \omega_{\mathrm{N} \gamma} r\right)^{\gamma} \exp \left(-r \omega_{\mathrm{N} \gamma}\right) L_{\mathrm{N}-1}^{2 \gamma+1}\left(2 \omega_{\mathrm{N} \gamma} r\right) \\
& g_{2}^{\mathrm{N} \kappa}(r)=N_{2}\left(2 \omega_{\mathrm{N} \gamma} r\right)^{\gamma-1} \exp \left(-r \omega_{\mathrm{N} \gamma}\right) L_{\mathrm{N}}^{2 \gamma-1}\left(2 \omega_{\mathrm{N} \gamma} r\right) .
\end{aligned}
$$

With $\omega_{\mathrm{N} \gamma}=\sqrt{\varepsilon_{0}^{2}-\varepsilon_{\mathrm{N} \gamma}^{2}}$ we note that

$$
\mathrm{N}=\alpha Z \varepsilon_{\mathrm{N} \gamma} / \omega_{\mathrm{N} \gamma}-\gamma
$$

is necessarily an integer, and this leads straight to the well known expression for the relativistic energy eigenvalues:

$$
E=m c^{2} /\left[1+\alpha^{2} Z^{2} /(\mathrm{N}+\gamma)^{2}\right]^{1 / 2} .
$$

(The negative root would render $\mathrm{N}$ negative and is therefore not applicable.) 
Some care is required in calculating the normalization constants, $N_{1}$ and $N_{2}$. Consideration of equation $(2.18 b)$ at $r=0$ leads to the following relationship between the two constants:

$$
\mathrm{N}(2 \gamma+\mathrm{N}) \omega_{\mathrm{N}_{y}} N_{1}=\left(\varepsilon_{\mathrm{N}_{\gamma}} \kappa+\varepsilon_{0} \gamma\right) N_{2} .
$$

Their absolute values are fixed by the normalization condition,

$$
\begin{aligned}
\int \psi_{E}(\boldsymbol{r})^{\dagger} \psi_{E} & (\boldsymbol{r}) \mathrm{d} \boldsymbol{r}=1 \\
& =\int_{0}^{\infty}\left[f_{1}(r)^{2}+f_{2}(r)^{2}\right] r^{2} \mathrm{~d} r \\
& =\frac{\kappa(\kappa+\gamma)}{2 \gamma^{2}} \int_{0}^{\infty}\left[g_{1}(r)^{2}+\frac{2 \alpha Z}{\kappa} g_{1}(r) g_{2}(r)+g_{2}(r)^{2}\right] r^{2} \mathrm{~d} r .
\end{aligned}
$$

The general integrals implicit in (2.26) are calculated in appendix 2 . Thus, substituting our solutions for $g_{1}$ and $g_{2}$ into (2.26) gives

$\frac{\kappa(\kappa+\gamma) \Gamma(2 \gamma+\mathbf{N})}{8 \gamma^{2} \mathrm{~N} ! \omega_{\mathrm{N \gamma}}}\left[(\gamma+\mathbf{N})(2 \gamma+\mathbf{N}) \mathbf{N} N_{1}^{2}-\frac{2 \alpha Z}{\kappa}(2 \gamma+\mathbf{N}) \mathbf{N} N_{1} N_{2}+(\gamma+\mathbf{N}) N_{2}^{2}\right]=1$.

Writing $N_{1}$ in terms of $N_{2}$, and eliminating $N$ from the term in square brackets noting (2.23) and

$$
\mathrm{N}(\mathrm{N}+2 \gamma)=\left(\kappa \varepsilon_{\mathrm{N} \gamma}-\gamma \varepsilon_{0}\right)\left(\kappa \varepsilon_{\mathrm{N} \gamma}+\gamma \varepsilon_{0}\right) / \omega_{\mathrm{N} \gamma}^{2}
$$

leads us finally to

$$
\begin{aligned}
& N_{2}^{\mathrm{N} \kappa}=2 \omega_{\mathrm{N} \gamma}^{2} /\left(\varepsilon_{0} \sqrt{\alpha Z}\right)\left[\mathrm{N} !\left(\varepsilon_{\mathrm{N} \gamma} \kappa-\gamma \varepsilon_{0}\right) /(\Gamma(2 \gamma+\mathrm{N})(\kappa+\gamma))\right]^{1 / 2} \\
& N_{1}^{\mathrm{N} \kappa}=\frac{\kappa}{|\kappa|} 2 \omega_{\mathrm{N} \gamma}^{2} /\left(\varepsilon_{0} \sqrt{\alpha Z}\right)\left[(\mathrm{N}-1) !\left(\kappa \varepsilon_{\mathrm{N} \gamma}+\gamma \varepsilon_{0}\right) /(\Gamma(2 \gamma+\mathrm{N}+1)(\kappa+\gamma))\right]^{1 / 2} .
\end{aligned}
$$

The solution when $\mathrm{N}=0$ requires special consideration. Clearly $N_{\mathrm{t}}$ must vanish otherwise the 'Laguerre polynomial' $L_{-1}^{2 \gamma+1}$, thought of as a hypergeometric function, will be non-terminating and therefore will not satisfy the boundary conditions. When $\mathrm{N}=0$ the energy is given by $\varepsilon_{0 \gamma}=\varepsilon_{0} \gamma /|\kappa|$ and consequently $\left(\varepsilon_{0 \gamma} \kappa+\varepsilon_{0} \gamma\right)=$ $\varepsilon_{0} \gamma(\kappa+|\kappa|) /|\kappa|$. Thus for positive $\kappa$, equation (2.25) implies that $N_{2}=0$ and the eigenfunction vanishes identically. In this case then, only negative values of $\kappa$ are allowed. The normalization constants are then given by

$$
\begin{aligned}
& N_{1}^{0 \gamma}=0 \\
& N_{2}^{0 \gamma}=4 \gamma\left(\varepsilon_{0} \alpha Z /|\kappa|\right)^{3 / 2} / \sqrt{\Gamma(2 \gamma+1) \kappa(\kappa+\gamma)} .
\end{aligned}
$$

This completes the solution of the Dirac-Coulomb equation.

It is instructive to examine more closely the relationship between the radial wavefunctions we have just computed, and the physical radial wavefunctions it was our original aim to derive. Noting that

$$
X=-(\kappa /|\kappa|) \sqrt{(\kappa-\gamma) /(\kappa+\gamma)} \quad 1-X^{2}=2 \gamma /(\kappa+\gamma)
$$


we can express (2.14) in terms of the total angular momentum $j$ and $\gamma$. Thus, when $\kappa=\left(j+\frac{1}{2}\right)$ we find

$$
\begin{aligned}
& f_{1}=\left(\sqrt{j+\frac{1}{2}+\gamma} g_{1}+\sqrt{j+\frac{1}{2}-\gamma} g_{2}\right) \sqrt{j+\frac{1}{2}+\gamma} / 2 \gamma \\
& f_{2}=\left(\sqrt{j+\frac{1}{2}-\gamma} g_{1}+\sqrt{j+\frac{1}{2}+\gamma} g_{2}\right) \sqrt{j+\frac{1}{2}+\gamma} / 2 \gamma
\end{aligned}
$$

and when $\kappa=-\left(j+\frac{1}{2}\right)$

$$
\begin{aligned}
& f_{1}=-\left(\sqrt{j+\frac{1}{2}-\gamma} g_{1}-\sqrt{j+\frac{1}{2}+\gamma} g_{2}\right) \sqrt{j+\frac{1}{2}-\gamma} / 2 \gamma \\
& f_{2}=\left(\sqrt{j+\frac{1}{2}+\gamma} g_{1}-\sqrt{j+\frac{1}{2}-\gamma} g_{2}\right) \sqrt{j+\frac{1}{2}-\gamma} / 2 \gamma .
\end{aligned}
$$

Notice that interchanging $g_{1}$ and $g_{2}$ in both cases simply interchanges $f_{1}$ and $f_{2}$. In view of the standard representations for the Dirac-Coulomb wavefunctions our result is seen to be in a particularly simple form. Perhaps the most interesting consequence of our solutions is that they admit an exceedingly straightforward treatment in the non-relativistic limit. In fact, in the limit as $\alpha Z \rightarrow 0$ only one or other of $g_{1}$ and $g_{2}$ contributes in equations (2.33), (2.34) and their contribution is transparently the Schrödinger-Coulomb wavefunction. This we will now demonstrate.

As is well known, the non-relativistic limit is equivalent to the limit as $\alpha Z \rightarrow 0$; it is important to note, however, that $\varepsilon_{0}$ is not independent of $\alpha Z$. In fact $\varepsilon_{0} \alpha Z=1 / a$. Thus the various factors occurring in the wavefunctions given to lowest order in $\alpha Z$ are $\omega_{\mathrm{N} \gamma}^{2} \simeq 1 / a(\mathrm{~N}+|\kappa|) \quad \gamma \simeq|\kappa| \quad|\kappa|+\gamma \simeq 2|\kappa| \quad|\kappa|-\gamma \approx \alpha^{2} Z^{2} /(2|\kappa|)$ $|\kappa| \varepsilon_{\mathrm{N} \gamma}+\gamma \varepsilon_{0} \simeq 2|\kappa| /(a \alpha Z) \quad|\kappa| \varepsilon_{\mathrm{N} \gamma}-\gamma \varepsilon_{0} \simeq \mathrm{N}(\mathrm{N}+2|\kappa|) \alpha Z /\left[2 a|\kappa|(\mathrm{N}+|\kappa|)^{2}\right]$ and $|\kappa|^{2}-\gamma^{2}=\alpha^{2} Z^{2}$ exactly.

For $\kappa=|\kappa|$, equations (2.33) become

$$
f_{1} \simeq g_{1}+\alpha Z / 2|\kappa| g_{2} \quad f_{2} \simeq \alpha Z / 2|\kappa| g_{1}+g_{2}
$$

and

$$
\begin{aligned}
& N_{1} \simeq 2 /\left[a^{3 / 2}(\mathrm{~N}+|\kappa|)^{2}\right] \sqrt{(\mathrm{N}-1) ! /(2|\kappa|+\mathrm{N}) !} \\
& N_{2} \simeq \mathrm{N}(\mathrm{N}+2|\kappa|) /\left[a^{3 / 2}|\kappa|(\mathrm{N}+|\kappa|)^{3}\right] \sqrt{(\mathrm{N}-1) ! /(2|\kappa|+\mathrm{N}) !}(\alpha \mathrm{Z}) .
\end{aligned}
$$

Thus to lowest order

$f_{1}(r)=\sqrt{\frac{(N-1) !}{(2|\kappa|+\mathrm{N}) !}}\left(\frac{2 r}{a(\mathrm{~N}+|\kappa|)}\right)^{|\kappa|} \frac{2 \mathrm{e}^{-r / a(\mathrm{~N}+|\kappa|)}}{a^{3 / 2}(\mathrm{~N}+|\kappa|)^{2}} L_{\mathrm{N}-1}^{2|\kappa|+1}\left(\frac{2 r}{a(\mathrm{~N}+|\kappa|)}\right)$

$f_{2}(r)=0$.

Examination of the angular part of the wavefunctions shows that we may put $|\kappa|=l$, and $\mathrm{N}=n-l$ where $n$ and $l$ are the non-relativistic quantum numbers. Thus, in the non-relativistic limit $f_{1}(r)=R_{n l}(r)$ and $f_{2}(r)=0$, as we would expect.

In the case when $\kappa=-|\kappa|$ we proceed in an exactly similar manner. This time $g_{2}$ survives:

$f_{1}=-\alpha^{2} Z^{2} / 4|\kappa|^{2} g_{1}+\alpha Z / 2|\kappa| g_{2} \quad f_{2}=\alpha Z / 2|\kappa| g_{1}-\alpha^{2} Z^{2} / 4|\kappa|^{2} g_{2}$

leads immediately to

$f_{1}(r)=\sqrt{\frac{\mathrm{N} !}{(2|\kappa|+\mathrm{N}-1) !}}\left(\frac{2 r}{a(\mathrm{~N}+|\kappa|)}\right)^{|\kappa|-1} \frac{2 \mathrm{e}^{-r / a(\mathrm{~N}+|\kappa|)}}{a^{3 / 2}(\mathrm{~N}+|\kappa|)^{2}} L_{\mathrm{N}}^{2 j \kappa \mid-1}\left(\frac{2 r}{a(\mathrm{~N}+|\kappa|)}\right)$

$f_{2}(r)=0$.

Putting now $|\kappa|=l+1$ and $N=n-l-1$ gives the expected result once again: $f_{1}(r)=$ $R_{n t}(r)$ and $f_{2}(r)=0$. 
This now completes our brief, if unconventional survey of the Dirac-Coulomb equation. The solutions given above have been derived before in what seems to us to be less natural treatments. The method is related to the treatment utilizing the secondorder Dirac equation [8]; the latter method treats the entire equation, however, whereas we have shown that the radial equations are all that we have to solve in a non-standard manner. Biedenharn [9] and later Wong and Yeh [10] were able to simplify the second-order approach somewhat, and Su [11] solved the ordinary Dirac equation in a non-standard manner, but both of these treatments involve the entire Dirac equation. The preceding solution apparently noticed first by Infeld [8] and rediscovered by us in a slightly different form [1] seems to be preferable in view of its manifest transparency.

\section{Recursion relations and other properties of the Coulomb radial wavefunctions.}

In this section we present a derivation of various recursion relations of the Coulomb radial wavefunctions given in the form of raising and lowering operators of the 'angular momentum' and 'principal' quantum numbers. Since we actually give the relations for the general radial functions defined in appendix 1 , our results will be applicable to both the non-relativistic and relativistic radial functions, as well as to a wider class of functions involving the Laguerre polynomials. All of the relations presented here will be required in the subsequent papers [3, 4]. Our analysis is performed on the Laplace transforms of the functions, which reduces much of the work involved to straightforward algebraic manipulations. We thus have a remarkably simply proof of the recursion relations of interest in and of itself.

In appendix 1 we show that the Laplace transform, $\mathscr{L}\left[r^{\lambda+1} f_{\nu \lambda}(r, \omega)\right](p)$, where

$$
\hat{f}_{\nu \lambda}(r, \omega)=(\nu-\lambda-\hat{1}) ! / \Gamma(\nu+\lambda+\hat{1}) r^{\lambda} \mathrm{e}^{-r_{\omega}} L_{\nu-\lambda-1}^{2 \lambda+1}(2 r \omega)
$$

is

$$
S_{\nu \lambda}(p ; \omega)=\left(\frac{p-\omega}{p+\omega}\right)^{\nu} \frac{1}{\left(p^{2}-\omega^{2}\right)^{\lambda+1}}
$$

and satisfies

$$
\left(\left(p^{2}-\omega^{2}\right) \frac{\mathrm{d}}{\mathrm{d} p}+2 p(\lambda+1)-2 \omega \nu\right) S_{\nu \lambda}(p ; \omega)=0 .
$$

The particularly simple form of $S$ allows us to quickly derive various raising and lowering operators for $\lambda$ and $\nu$ which will, in turn, lead to raising and lowering operators of $f$.

From (3.2) we see immediately that

$$
\left(p^{2}-\omega^{2}\right) S_{\nu \lambda}(p ; \omega)=S_{\nu \lambda-1}(p ; \omega)
$$

which is a lowering operator for $\lambda$. Let us now differentiate (3.4) with respect to $p$. Then

$$
\begin{aligned}
\frac{\mathrm{d}}{\mathrm{d} p} S_{\nu \lambda-1}(p ; \omega) & =\frac{\mathrm{d}}{\mathrm{d} p}\left(\left(p^{2}-\omega^{2}\right) S_{\nu^{\prime \lambda}}(p ; \omega)\right) \\
& =\left(p^{2}-\omega^{2}\right) \frac{\mathrm{d}}{\mathrm{d} p} S_{\nu_{\lambda}}(p ; \omega)+2 p S_{\nu \lambda}(p ; \omega) \\
& =[2 \omega \nu-2 p(\lambda+1)] S_{v \lambda}(p ; \omega)+2 p S_{i \lambda}(p ; \omega) \\
& =-2(\lambda p-\omega \nu) S_{\nu \lambda}(p ; \omega)
\end{aligned}
$$


where we have made use of (3.3) at the third step. Now, taking note of (3.4) again we have

$$
\begin{aligned}
(\lambda p+\omega \nu) \frac{\mathrm{d}}{\mathrm{d} p} & S_{\nu \lambda-1}(p ; \omega) \\
& =-2\left[\lambda^{2} p^{2}-(\omega \nu)^{2}\right] S_{\nu \lambda}(p ; \omega) \\
& =-2 \lambda^{2}\left[\omega^{2} S_{\nu \lambda}(p ; \omega)+S_{\nu \lambda-1}(p ; \omega)\right]+2(\omega \nu)^{2} S_{\nu \lambda}(p ; \omega)
\end{aligned}
$$

giving finally the following raising operator for $\lambda$ :

$$
\left[(\lambda p+\omega \nu) \frac{\mathrm{d}}{\mathrm{d} p}+2 \lambda^{2}\right] S_{\nu \lambda-1}(p ; \omega)=2(\omega \nu)^{2}\left(\frac{\nu^{2}-\lambda^{2}}{\nu^{2}}\right) S_{\nu \lambda}(p ; \omega) .
$$

The raising and lowering operators for $\nu$ are only slightly more complicated. From (3.2) we see that

$$
(p+\omega) S_{\nu \lambda}(p ; \omega)=\left(\frac{\nu}{\nu-1}\right)^{2 \lambda+2}(p-\omega) S_{\nu-1 \lambda}\left(\frac{\nu}{\nu-1} p ; \frac{\nu}{\nu-1} \omega\right)
$$

and this equation, together with (3.4), implies

$$
\begin{aligned}
& S_{\nu \lambda-1}(p ; \omega)=\left(\frac{\nu}{\nu-1}\right)^{2 \lambda+2}(p-\omega)^{2} S_{\nu-1 \lambda}\left(\frac{\nu}{\nu-1} p ; \frac{\nu}{\nu-1} \omega\right) \\
& (p+\omega)^{2} S_{\nu \lambda}(p ; \omega)=\left(\frac{\nu}{\nu-1}\right)^{2 \lambda} S_{\nu-1 \lambda-1}\left(\frac{\nu}{\nu-1} p ; \frac{\nu}{\nu-1} \omega\right) .
\end{aligned}
$$

The plan is now to raise the $\lambda-1$ to $\lambda$ in (3.8) and (3.9) making use of (3.6), this being facilitated by the fact that the differential operator does not depend on $\nu$. Beginning with (3.8) we find

$$
\begin{aligned}
2 \omega \nu\left(\frac{\nu^{2}-\lambda^{2}}{\nu^{2}}\right) & S_{\nu \lambda}(p ; \omega) \\
= & \left(\frac{\nu}{\nu-1}\right)^{2 \lambda+2}\left[(p-\omega)^{2}\left((p+\omega \nu) \frac{\mathrm{d}}{\mathrm{d} p}+2 \lambda^{2}\right)+2(p-\omega)(\lambda p+\omega \nu)\right] \\
& \times S_{\nu-\mid \lambda}\left(\frac{\nu}{\nu-1} p ; \frac{\nu}{\nu-1} \omega\right) .
\end{aligned}
$$

We now make repeated use of (3.3) to reduce terms in $p^{3} \mathrm{~d} / \mathrm{d} p$ and $p^{2} \mathrm{~d} / \mathrm{d} p$ to terms at most of the form $p \mathrm{~d} / \mathrm{d} p$. Then we arrive at a $\nu$ raising operator:

$$
\left((\nu-\lambda-1)-(p-\omega) \frac{\mathrm{d}}{\mathrm{d} p}\right) S_{\nu \lambda}(p ; \omega)=(\nu+\lambda+1)\left(\frac{\nu}{\nu+1}\right)^{2 \lambda+2} S_{\nu+1 \lambda}\left(\frac{\nu}{\nu+1} p ; \frac{\nu}{\nu+1} \omega\right) \text {. }
$$

The same procedure applied to (3.9) gives a $\nu$ lowering operator just as quickly:

$$
\left((\nu+\lambda+1)+(p+\omega) \frac{\mathrm{d}}{\mathrm{d} p}\right) S_{\nu \lambda}(p ; \omega)=(\nu-\lambda-1)\left(\frac{\nu}{\nu-1}\right)^{2 \lambda+2} S_{\nu^{\prime \prime-1 \lambda}}\left(\frac{\nu}{\nu-1} p ; \frac{\nu}{\nu-1} \omega\right) \text {. }
$$


Both (3.10) and (3.11) can be checked directly given the simple algebraic form of $S$.

The recursion relations for $f_{\nu \lambda}$ are given by the inverse Laplace transforms of (3.5), (3.6), (3.10) and (3.11) respectively:

$\left[\lambda\left(\frac{\mathrm{d}}{\mathrm{d} r}+\frac{\lambda+1}{r}\right)-\omega \nu\right] f_{\nu \lambda}(r, \omega)=\frac{1}{2} f_{\nu \lambda-1}(r, \omega)$

$\left[\lambda\left(\frac{\mathrm{d}}{\mathrm{d} r}-\frac{\lambda-1}{r}\right)+\omega \nu\right] f_{\nu \lambda-1}(r, \omega)=-2(\omega \nu)^{2}\left(\frac{\nu^{2}-\lambda^{2}}{\nu^{2}}\right) f_{\nu \lambda}(r, \omega)$

$\left[(\nu+1)+r\left(\frac{\mathrm{d}}{\mathrm{d} r}-\omega\right)\right] f_{\nu \lambda}(r, \omega)=(\nu+\lambda+1)\left(\frac{\nu}{\nu+1}\right)^{\lambda} f_{\nu+1 \lambda}\left(\frac{\nu+1}{\nu} r, \frac{\nu}{\nu+1} \omega\right)$

$\left[(\nu-1)-r\left(\frac{\mathrm{d}}{\mathrm{d} r}+\omega\right)\right] f_{\nu \lambda}(r, \omega)=(\nu-\lambda-1)\left(\frac{\nu}{\nu-1}\right)^{\lambda} f_{\nu-1 \lambda}\left(\frac{\nu-1}{\nu} r, \frac{\nu}{\nu-1} \omega\right)$.

We can now apply these ladder operators to the Schrödinger-Coulomb wavefunctions and to the Dirac-Coulomb wavefunctions. We begin with the radial SchrödingerCoulomb wavefunctions. Since

$$
f_{n l}(r, 1 / a n)=(-1)^{n-1-1}\left(n^{2} a^{3 / 2}\right) / 2(a n / 2)^{l} \sqrt{(n-l-1) ! /(n+l) !} R_{n l}(r)
$$

we have the following relationships $[13]$ :

$\left[l\left(\frac{\mathrm{d}}{\mathrm{d} r}+\frac{l+1}{r}\right)-1 / a\right] R_{n l}(r)=-1 / a \sqrt{1-l^{2} / n^{2}} R_{n l-1}(r)$

$\left[l\left(\frac{\mathrm{d}}{\mathrm{d} r}-\frac{l-1}{r}\right)+1 / a\right] R_{n l-1}(r)=1 / a \sqrt{1-l^{2} / n^{2}} R_{n i}(r)$

$\left[(n+1)+r\left(\frac{\mathrm{d}}{\mathrm{d} r}-1 /(a n)\right)\right] R_{n l}(r)=\left(\frac{n+1}{n}\right)^{2} \sqrt{(n-1)(n+1+1)} R_{n+1 /}\left(\frac{n+1}{n} r\right)$

$\left[(n-1)-r\left(\frac{\mathrm{d}}{\mathrm{d} r}+1 /(a n)\right)\right] R_{n l}(r)=\left(\frac{n-1}{n}\right)^{2} \sqrt{(n+l)(n-l-1)} R_{n-11}\left(\frac{n-1}{n} r\right)$.

In the relativistic case we have to be clear to distinguish normalization constants for different values of $\mathrm{N}$. We begin by noting that

$$
\begin{aligned}
& f_{\mathrm{N}+\gamma, \gamma}\left(r, \omega_{\mathrm{N \gamma}}\right)=\frac{(\mathrm{N}-1) !}{\Gamma(2 \gamma+\mathrm{N}+1)} 1 /\left[N_{1}^{\mathrm{N} \kappa}\left(2 \omega_{\mathrm{N} \gamma}\right)^{\gamma}\right] g_{1}^{\mathrm{N} \kappa}(r) \\
& f_{\mathrm{N}+\gamma, \gamma-1}\left(r, \omega_{\mathrm{N} \gamma}\right)=\frac{\mathrm{N} !}{\Gamma(2 \gamma+\mathrm{N})} 1 /\left[N_{2}^{\mathrm{N} \kappa}\left(2 \omega_{\mathrm{N} \gamma}\right)^{\gamma-1}\right] g_{2}^{\mathrm{N} \kappa}(r)
\end{aligned}
$$

where $\omega_{N \gamma}=\sqrt{\varepsilon_{0}^{2}-\varepsilon_{N \gamma}^{2}}$ and from which, noting (2.25), we find that (3.12) becomes

$$
\left[\gamma\left(\frac{\mathrm{d}}{\mathrm{d} r}+\frac{\gamma+1}{r}\right)-\alpha Z \varepsilon_{\mathrm{N} \gamma}\right] g_{1}^{\mathrm{N} \kappa}(r)=\left(\varepsilon_{\mathrm{N} \gamma} \kappa+\varepsilon_{0} \gamma\right) g_{2}^{\mathrm{N} \kappa}(r)
$$

which is just equation $(2.18 b)$, and (3.13) becomes

$$
\left[\gamma\left(\frac{\mathrm{d}}{\mathrm{d} r}-\frac{\gamma-1}{r}\right)+\alpha Z \varepsilon_{\mathrm{N} \gamma}\right] g_{2}^{\mathrm{N} \kappa}(r)=-\left(\varepsilon_{\mathrm{N} \gamma} \kappa-\varepsilon_{0} \gamma\right) g_{1}^{\mathrm{N}^{*}}(r)
$$

which is equation $(2.18 a)$. Notice that the $\lambda$ raising and lowering operators do not change $\kappa$, which appears under a root sign in $\gamma$ and is thus inaccessible; rather they interchange the roles of $g_{1}$ and $g_{2}$. These recursion relations were first noted by Infeld [7] and Biedenharn [9]. 
The $\nu$ raising and lowering operators appear in a somewhat more complicated form. We content ourselves with presenting the results, which are new:

$$
\begin{aligned}
& {\left[(\mathrm{N}+\gamma+1)+r\left(\frac{\mathrm{d}}{\mathrm{d} r}-\omega_{\mathrm{N} \gamma}\right)\right] g_{1}^{\mathrm{N} \kappa}(r)=\sqrt{\mathrm{N}(\mathrm{N}+1+2 \gamma)} B_{\mathrm{N} \kappa}^{2} D_{\mathrm{N} \kappa} g_{1}^{\mathrm{N}+1 \kappa}\left(r C_{\mathrm{N} \kappa}\right)} \\
& {\left[(\mathrm{N}+\gamma+1)+r\left(\frac{\mathrm{d}}{\mathrm{d} r}-\omega_{\mathrm{N} \gamma}\right)\right] g_{2}^{\mathrm{N} \kappa}(r)} \\
& =(\mathrm{N}+2 \gamma) \sqrt{\mathrm{N} /(\mathrm{N}+1+2 \gamma)} B_{\mathrm{N} \kappa}^{3} / D_{\mathrm{N} \kappa} g_{2}^{\mathrm{N}+1 \kappa}\left(r C_{\mathrm{N} \kappa}\right) \\
& {\left[(\mathrm{N}+\gamma-1)-r\left(\frac{\mathrm{d}}{\mathrm{d} r}+\omega_{\mathrm{N} \gamma}\right)\right] g_{1}^{\mathrm{N} \kappa}(r)} \\
& \quad=\sqrt{(\mathrm{N}-1)(\mathrm{N}+2 \gamma)} 1 /\left(B_{\mathrm{N}-1 \kappa}^{2} D_{\mathrm{N}-1 \kappa}\right) g_{1}^{\mathrm{N}-1 \kappa}\left(r / C_{\mathrm{N}-1 \kappa}\right) \\
& {\left[(\mathrm{N}+\gamma-1)-r\left(\frac{\mathrm{d}}{\mathrm{d} r}+\omega_{\mathrm{N} \gamma}\right)\right] g_{2}^{\mathrm{N} \kappa}(r)} \\
& =\mathrm{N} \sqrt{(\mathrm{N}+2 \gamma) /(\mathrm{N}-1)} D_{\mathrm{N}-1 \kappa} / B_{\mathrm{N}-1 \kappa}^{3} g_{2}^{\mathrm{N}-1 \kappa}\left(r / C_{\mathrm{N}-1 \kappa}\right)
\end{aligned}
$$

where

$$
B_{\mathrm{N} \kappa}=\left(\varepsilon_{\mathrm{N} \gamma} / \varepsilon_{\mathrm{N}+1 \gamma}\right)(\mathrm{N}+1+\gamma) /(\mathrm{N}+\gamma) \quad C_{\mathrm{N} \kappa}=\omega_{\mathrm{N} \gamma} / \omega_{\mathrm{N}+1 \gamma}
$$

and

$$
D_{\mathrm{N} \kappa}=\left[\left(\kappa \varepsilon_{\mathrm{N} \gamma}+\gamma \varepsilon_{0}\right) /\left(\kappa \varepsilon_{\mathrm{N}+1 \gamma}+\gamma \varepsilon_{0}\right)\right]^{1 / 2} .
$$

It is interesting to note that the last four recursion relations can easily be shown to reduce to the non-relativistic ones in the limit $\alpha Z \rightarrow 0$.

We end this section with presenting some results which will be of use in the papers on the Green functions. By considering the function

$$
f_{k+\lambda+1, \lambda}(r, \omega)=\frac{k !}{\Gamma(2 \lambda+2+k)} r^{\lambda} \mathrm{e}^{-r \omega} L_{k}^{2 \lambda+1}(2 \omega r)
$$

where $k$ is an integer, and rearranging equations (3.11), (3.12), (3.13), we can derive the following formulae:

$$
\begin{aligned}
& {\left[\frac{\mathrm{d}^{2}}{\mathrm{~d} r^{2}}+\frac{2}{r} \frac{\mathrm{d}}{\mathrm{d} r}-\frac{\lambda(\lambda+1)}{r^{2}}+\frac{2 \omega \nu}{r}-\omega^{2}\right]\left(r^{\lambda} \mathrm{e}^{-r \omega} L_{k}^{2 \lambda+1}(2 \omega r)\right)} \\
& =-2 \omega(k+\lambda+1-\nu) r^{\lambda-1} \mathrm{e}^{-r \omega} L_{k}^{2 \lambda+1}(2 \omega r) \\
& {\left[\lambda\left(\frac{\mathrm{d}}{\mathrm{d} r}+\frac{\lambda+1}{r}\right)-\omega \nu\right]\left(r^{\lambda} \mathrm{e}^{-r \omega} L_{k}^{2 \lambda+1}(2 \omega r)\right)} \\
& =(k+\lambda+1-\nu) \omega r^{\lambda} \mathrm{e}^{-r \omega} L_{k}^{2 \lambda+1}(2 \omega r) \\
& +(k+1)(2 \lambda+k+1) / 2 r^{\lambda-1} \mathrm{e}^{-r \omega} L_{k+1}^{2 \lambda-1}(2 \omega r) \\
& {\left[\lambda\left(\frac{\mathrm{d}}{\mathrm{d} r}-\frac{\lambda-1}{r}\right)+\omega \nu\right]\left(r^{\lambda-1} \mathrm{e}^{-r \omega} L_{k}^{2 \lambda-1}(2 \omega r)\right)} \\
& =-(k+\lambda-\nu) \omega r^{\lambda-1} \mathrm{e}^{-r \omega} L_{k}^{2 \lambda-1}(2 \omega r) \\
& -2 \omega^{2}\left\{1-\delta_{k 0}\right\} r^{\lambda} \mathrm{e}^{-r \omega} L_{k-1}^{2 \lambda+1}(2 \omega r) .
\end{aligned}
$$

These last two general $\lambda$ raising and lowering operators will be extensively employed in our analysis of the reduced Green functions [4]. 


\section{Acknowledgments}

Research support by the Natural Sciences and Engineering Research Council of Canada is gratefully acknowledged.

\section{Appendix 1. Solution of the generalized radial equation}

In this appendix we use the method of Laplace transforms to solve the general radial Schrödinger-Coulomb type equation which arises in our solution of both the nonrelativistic and the relativistic Coulomb problem in section 2. Apart from its novelty, this method is easily generalized to allow for the solution of the corresponding general radial equation satisfied by the non-relativistic and transformed relativistic Green functions [3]. This latter equation is complicated by the presence of a delta function which, nonetheless, becomes quite manageable in the Laplace transformed space. The use of Laplace transform methods to derive the radial SCWF is not in itself new: indeed such techniques were used by Schrödinger in his first paper on the quantum mechanical hydrogen atom [14].

Specifically, in this appendix we prove that the solution of

$$
\left[\frac{\mathrm{d}^{2}}{\mathrm{~d} r^{2}}+\frac{2}{r} \frac{\mathrm{d}}{\mathrm{d} r}-\frac{\lambda(\lambda+1)}{r^{2}}+\frac{2 \omega \nu}{r}-\omega^{2}\right] f(r)=0
$$

(with $\lambda, \omega$ and $\nu$ positive) subject to the boundary conditions

$$
r f \in \mathrm{L}^{2}(\mathbb{R}) \quad \text { and } \quad \lim _{r \rightarrow 0} r f(r)=0
$$

is

$$
f(r)=N r^{\lambda} \mathrm{e}^{-r \omega} L_{\nu-\lambda-1}^{2 \lambda+1}(2 \omega r)
$$

with $\nu-\lambda-1$ a non-negative integer, and $N$ an arbitrary constant. Here $L_{n}^{\alpha}$ is a generalized Laguerre polynomial as defined in appendix 3.

We begin our proof by considering a related function,

$$
h(r)=r^{\lambda+1} f(r)
$$

which satisfies

$$
\left(r \frac{\mathrm{d}^{2}}{\mathrm{~d} r^{2}}-2 \lambda \frac{\mathrm{d}}{\mathrm{d} r}+2 \omega \nu-r \omega^{2}\right) h(r)=0 .
$$

The Laplace transform of $h$,

$$
H(p)=\int_{0}^{\infty} \mathrm{e}^{-p r} h(r) \mathrm{d} r
$$

satisfies the transformed equation

$$
\left(\left(p^{2}-\omega^{2}\right) \frac{\mathrm{d}}{\mathrm{d} p}+2 p(\lambda+1)-2 \omega \nu\right) H(p)=0
$$

where we have used the fact that $h(0)=0$. Finally, the function

$$
G(p)=p^{2 \lambda+2} H(p)
$$


satisfies

$$
\left(\left(p^{2}-\omega^{2}\right) \frac{\mathrm{d}}{\mathrm{d} p}+\frac{2(\lambda+1) \omega^{2}}{p}-2 \omega \nu\right) G(p)=0 .
$$

This equation is most easily solved if we make the change of variables

$$
p \mapsto g_{p} \risingdotseq(\dot{p}-\omega) / 2 p
$$

so that

$$
\left(q(1-q) \frac{\mathrm{d}}{\mathrm{d} q}+(\lambda+1)(1-2 q)-\nu\right) G(q)=0
$$

which has the simple solution

$$
G(q)=N^{\prime \prime} q^{\nu-\lambda-1}(1-q)^{-r-\lambda-1} .
$$

Thus the Laplace transform of $h$ is given by

$$
H(p)=N^{\prime}\left(\frac{p-\omega}{p+\omega}\right)^{\nu}[(p-\omega)(p+\omega)]^{-\lambda-1} .
$$

$N^{\prime}$ and $N^{\prime \prime}$ are arbitrary constants.

We can immediately deduce a condition on the magnitude of $\nu$. If $\nu-\lambda-1<0$, then the singularity of $H(p)$ with greatest real part is at $p=\omega$, and thus $h(r)$ (and consequently $f(r)$ ) will behave as $\mathrm{e}^{r e s}$ as $r$ approaches infinity [15]. Since this would violate the integrability condition on $f$, we deduce that $\nu \geqslant \lambda+1$. Now

$$
\begin{aligned}
H(p) & =N^{\prime}\left(1-\frac{2 \omega}{p+\omega}\right)^{\nu-\lambda-1}(p+\omega)^{-2 \lambda-2} \\
& =N^{\prime} \sum_{n=0}^{\infty} \frac{(\lambda+1-\nu)_{n}}{n !}(2 \omega)^{n}(p+\omega)^{-2 \lambda-2-n}
\end{aligned}
$$

a series which converges absolutely for $p>\omega$. Applying a simple extension of a theorem on Laplace transforms [16] we can immediately deduce that

$$
h(r)=N r^{2 \lambda+1} \mathrm{e}^{-r \omega} \sum_{n=0}^{\infty} \frac{(\lambda+1-\nu)_{n}}{n ! \Gamma(2 \lambda+2+n)}(2 \omega r)^{n} .
$$

Familiar arguments now can be used to show that $h(r)$ behaves like $\mathrm{e}^{r \omega}$ at infinity and is therefore not square integrable unless $\lambda+1-\nu$ is a negative integer. In view of the definition of Laguerre polynomials given in appendix 3 the form of $f$ given in the theorem follows.

\section{Appendix 2. Calculation of some integrals required for the normalization of wavefunctions}

Several methods of obtaining these integrals are known [12]. However, the following are simple proofs requiring only a knowledge of Vandermonde's theorem (appendix 3 ). 
(i)

$$
\begin{aligned}
\int_{0}^{\infty} \mathrm{e}^{-x} x^{\alpha}{ }_{1} & F_{1}(-n ; \alpha ; x)^{2} \mathrm{~d} x \\
& =\sum_{i=0}^{n} \sum_{j=0}^{n}\left(\begin{array}{l}
n \\
i
\end{array}\right)\left(\begin{array}{l}
n \\
j
\end{array}\right)(-1)^{i+j} \frac{\Gamma(\alpha)^{2}}{\Gamma(\alpha+i) \Gamma(\alpha+j)} \int_{0}^{\infty} \mathrm{e}^{-x} x^{\alpha+i+j} \mathrm{~d} x \\
& =\sum_{i=0}^{n}\left(\begin{array}{l}
n \\
i
\end{array}\right)(-1)^{i} \frac{\Gamma(\alpha) \Gamma(\alpha+i+1)}{\Gamma(\alpha+i)}{ }_{2} F_{1}(-n, \alpha+i+1 ; \alpha ; 1) \\
& =\sum_{i=0}^{n}\left(\begin{array}{l}
n \\
i
\end{array}\right)(-1)^{i} \frac{\Gamma(\alpha) \Gamma(\alpha+i+1)}{\Gamma(\alpha+i)}(-i-1)_{n} /(\alpha)_{n} \\
& =\frac{\Gamma(\alpha)^{2}(\alpha+2 n)}{\Gamma(\alpha+n)} n !
\end{aligned}
$$

Hence

$$
\int_{0}^{\infty} \mathrm{e}^{-x} x^{\alpha}\left[L_{n}^{\alpha-1}(x)\right]^{2} \mathrm{~d} x=(\alpha+2 n) \Gamma(\alpha+n) / n !
$$

(ii)

$$
\begin{aligned}
& \int_{0}^{\infty} \mathrm{e}^{-x} x^{\alpha+1}{ }_{1} F_{1}(-n ; \alpha ; x)_{1} F_{1}(-n+1 ; \alpha+2 ; x) \mathrm{d} x=-2 \frac{\Gamma(\alpha) \Gamma(\alpha+2)}{\Gamma(\alpha+n)} n ! \\
& \int_{0}^{\infty} \mathrm{e}^{-x} x^{\alpha+1} L_{n}^{\alpha-1}(x) L_{n-1}^{\alpha+1}(x) \mathrm{d} x=-2 \Gamma(\alpha+n+1) / n ! .
\end{aligned}
$$

(iii)

$$
\begin{aligned}
& \int_{0}^{\infty} \mathrm{e}^{-x} x^{\alpha}{ }_{1} F_{1}(-n ; \alpha+1 ; x)^{2} \mathrm{~d} x=\frac{\Gamma(\alpha+1)^{2}}{\Gamma(\alpha+1+n)} n ! \\
& \int_{0}^{\infty} \mathrm{e}^{-x} x^{\alpha}\left[L_{n}^{\alpha}(x)\right]^{2} \mathrm{~d} x=\Gamma(\alpha+n+1) / n ! .
\end{aligned}
$$

The last two Laplace transforms were derived in exactly the same manner as the first, by expanding the confluent hypergeometric functions, integrating, and using Vandermonde's theorem once.

\section{Appendix 3. Notation and special functions}

Hypergeometric functions

$$
\begin{aligned}
& (\alpha)_{n}=\Gamma(\alpha+n) / \Gamma(\alpha) \\
& { }_{1} F_{1}(\alpha ; \beta ; x)=\sum \frac{(\alpha)_{n}}{(\beta)_{n}} \frac{x^{n}}{n !} \\
& { }_{2} F_{1}(\alpha, \beta ; \gamma, x)=\sum \frac{(\alpha)_{n}(\beta)_{n}}{(\gamma)_{n}} \frac{x^{n}}{n !} .
\end{aligned}
$$

Vandermonde's theorem

$$
{ }_{2} F_{1}(-n, b ; c ; 1)=(c-b)_{n} /(c)_{n} .
$$


Laguerre polynomials

$$
L_{n}^{\alpha}(x)=\frac{\Gamma(\alpha+1+n)}{\Gamma(\alpha+1) n !}{ }_{1} F_{1}(-n ; \alpha+1 ; x) .
$$

Dirac matrices

$$
\begin{array}{ll}
\boldsymbol{\alpha}=\left(\begin{array}{ll}
0 & \boldsymbol{\sigma} \\
\boldsymbol{\sigma} & 0
\end{array}\right) & \beta=\left(\begin{array}{rr}
1 & 0 \\
0 & -1
\end{array}\right) \quad \boldsymbol{\sigma}=\left[\left(\begin{array}{ll}
0 & 1 \\
1 & 0
\end{array}\right),\left(\begin{array}{rr}
0 & -i \\
i & 0
\end{array}\right),\left(\begin{array}{rr}
1 & 0 \\
0 & -1
\end{array}\right)\right] \\
\mathbf{\Sigma} & =\left(\begin{array}{ll}
\boldsymbol{\sigma} & 0 \\
0 & \boldsymbol{\sigma}
\end{array}\right) .
\end{array}
$$

\section{References}

[1] Swainson R A $1988 P h D$ Thesis University of Windsor

[2] Goldman S P and Drake G W F 1981 Phys, Rev. A 24183

[3] Swainson R A and Drake G W F J. Phys. A: Math. Gen. 23

[4] Swainson R A and Drake G W F J. Phys. A: Math. Gen. to be submitted

[5] Edmonds A R 1957 Angular Momentum in Quantum Mechanics (Princeton, NJ: Princeton University Press)

[6] Bethe H A and Salpeter E E 1977 Quantum Mechanics of One-and Two-Electron Atoms (New York: Plenum)

[7] Infeld L 1941 Phys. Rev. 59737

[8] Martin P C and Glauber R J 1958 Phys. Rev. 1091307

[9] Biedenharn L C 1962 Phys. Rev. 126845

[10] Wong M K F and Yeh H Y 1982 Phys. Rev. D 253396

[11] Su J Y 1985 Phys. Rev. A 323251

[12] Lin C C 1941 Phys. Rev. 59841

[13] Biedenharn L C and Louck J D 1981 Angular Momentum in Quantum Physics: Theory and Applications (Encyclopedia of Mathematics and its Applications 8) (Reading, MA: Addison-Wesley)

[14] Schrödinger E 1926 Ann. der Physik 79361

[15] Doetsch G 1961 Guide to the Applications of Laplace Transforms (Princeton, NJ: Van Nostrand)

[16] Le Page W R 1980 Complex Variables and the Laplace Transform for Engineers (New York: Dover) 